chen Titel 2008 veröffentlichte Dokumentation der „Schicksale jüdischer Anwälte in Deutschland nach 1933“ ergänzt. Keine Berücksichtigung fanden in Berlin als Syndikus arbeitende Anwälte, die andernorts zugelassen oder nicht in der Stadtgemeinde ansässig waren. Zu diesem Personenkreis zählt der Dortmunder Anwalt Max Frank, 1921 kurzzeitig Mitglied des Reichstags (SPD), der sich im Mai 1933 in Berlin das Leben genommen hat. Sein Name fehlt daher hier und bis heute im Gedenkbuch des Deutschen Bundestages.

Martin Schumacher

\title{
MITTEILUNG
}

\section{Förderpreise der Deutschen Vereinigung für Politische Wissenschaft}

Im Jahr 2009 schreibt die Deutsche Vereinigung für Politische Wissenschaft erneut zwei mit jeweils 1.000 Euro dotierte Förderpreise für politikwissenschaftliche Arbeiten aus. Ein Preis wird für eine Dissertation vergeben, ein weiterer für eine Monographie nach der Promotion. Folgende Bedingungen gelten:

(1) In Frage kommende Publikationen sollen 2008 veröffentlicht worden sein.

(2) Dissertationen müssen mindestens mit magna cum laude bewertet sein.

(3) Bewerberinnen und Bewerber dürfen zum Zeitpunkt der Bewerbung nicht zur Gruppe der Hochschullehrer und -lehrerinnen beziehungsweise Gleichgestellter in fester Anstellung gehören. Bewerbungen von Juniorprofessorinnen und -professoren sowie von Kolleginnen und Kollegen, die eine Professur befristet vertreten, sind willkommen.

(4) Der Kreis der Bewerber und Bewerberinnen ist auf Personen beschränkt, die in Deutschland promoviert wurden, oder Deutsche, die im Ausland promoviert wurden.

(5) Für die beiden Förderpreise sind Selbstbewerbungen möglich; selbstverständlich sind Vorschläge von Dritten willkommen. Verlage sind nicht vorschlagsberechtigt.

(6) Frauen sind besonders aufgefordert, sich zu bewerben; dies gilt auch für die Benennung von Kandidatinnen durch Dritte.

(7) Einsendefrist für 2008 veröffentlichte Publikationen ist der 28. Februar 2009.

Vorschläge und Benennungen für die Wissenschaftspreise sind zusammen mit vier Exemplaren der Veröffentlichung und einer Kopie der Promotionsurkunde an die Geschäftsstelle der DVPW zu senden: Deutsche Vereinigung für Politische Wissenschaft, c/o Universität Osnabrück, FB 1 - Sozialwissenschaften, D-49069 Osnabrück, www.dvpw.de.

Vorstand und Beirat werden die Vorschläge durch eine aus drei Kolleginnen und Kollegen bestehende Jury begutachten lassen. Die Publikationen werden nach Abschluss des Verfahrens bei den Jurymitgliedern und eventuell in Anspruch genommenen Gutachtern verbleiben. Um Kosten zu sparen, ist es ratsam, den publizierenden Verlag zu bitten, vier Freiexemplare zu übersenden. 